\title{
Density theorems for ideal points in vector optimization
}

\author{
A. Balbás ${ }^{\mathrm{a}, *}$, M. Ballvé ${ }^{\mathrm{b}}$, P. Jiménez Guerra ${ }^{\mathrm{b}}$ \\ ${ }^{a}$ Departamento de Economía de la Empresa, Universidad Carlos III, ClMadrid 126, 28903 Getafe, Madrid, Spain \\ b Departamento de Matemáticas Fundamentales, Facultad de Ciencias, UNED, ClSenda del Rey s/n 28040, Madrid, Spain
}

\begin{abstract}
Several results are established concerning the density of the set of ideal points in the set of minimal solutions of positive support functionals of sets in normed linear spaces. The above ideal points are defined and several charac terizations and sufficient conditions for their existence are also stated.
\end{abstract}

Keywords: Mathematical programming; Vector optimization; Efficient point; Density theorems; Normed linear spaces

\section{Introduction}

From the publication of the important result of Arrow et al. (1953), concerning the density of the minimal solutions of strictly positive support functionals in the set of minimal elements of compact convex subsets of $R^{n}$, a variety of density results have been provided in several settings and under different assumptions (see, for instance, Makarov and Rachkovski, 1996). In particular, many extensions of the above result of Arrow, Barankin and Blackwell have been given by several authors (see, for instance, Ferro, 1993; Gallagher and Saleh, 1993; Chen, 1995).

${ }^{*}$ Corresponding author.

E mail addresses: balbas@emp.uc3m.es (A. Balbás), mballve @mat.uned.es (M. Ballvé), pjimenez@mat.uned.es (P. Jiménez Guerra).
The present paper deals with the density of ideal points of a subset $D$ of an arbitrary normed linear space, partially ordered by a closed, convex and pointed cone, in the set of points in $D$ that can be supported by positive functionals. Since strictly positive functionals are obviously positive, interesting results concerning the density of ideal points of $D$ in the set of minimal points in $D$ can be obtained by combining the density results stated here with several existing extensions of the Arrow, Barankin and Blackwell theorem to normed linear spaces. This is especially interesting because ideal points are minima, and it is known that most properties in optimization are far more easily studied for minimum solutions than for minimal ones (see, for instance, Zowe, 1977). This is particularly clear in sensitivity analysis, where ideal points can be very useful (see Balbás et al., 1995; Balbás and Jiménez Guerra, 1996, 1997).

An element $x_{0}$ of a real normed vector space $X$, partially ordered by a closed, convex and pointed 
cone $C$, is said to be an ideal point of a set $D \subset X$ if there exists another closed, convex and pointed cone $K \subset X$ such that $C \subset K$ and $D \subset x_{0}+K$.

Proposition 2.2 states that $x_{0} \in X$ is an ideal point of $D$ if and only if there exists a real normed vector space $Y$, partially ordered by a closed, convex and pointed cone $S$, and a strictly positive topological isomorphism $T: X \rightarrow Y$ such that $T(D) \subset T\left(x_{0}\right)+S$. In many occasions, this characterization makes it easier to work in practice with ideal points (see Balbás and Jiménez Guerra, 1996).

Proposition 2.3 proves that a point $x_{0} \in X$ is an ideal point of $D$ if the set of all positive functionals supporting $x_{0}$ separates points of $X$, and Proposition 2.4 states that every point $x_{0} \in X$, such that 0 is a denting point of cone $\left(D-x_{0}\right)+C$, is an ideal point of $D$.

Section 3 is devoted to density theorems. In particular, Theorem 3.1 states that, if $D$ is a normbounded subset of $X$, then the set of ideal points in $D$ is dense in the set of points in $D$ that can be supported by positive functionals. As an immediate consequence, we have the fact that if the cone $C$ has non-empty interior, then the set of ideal points of a norm-bounded subset $D$ is dense in the set of all minimal points of $D$ (see Corollary 3.2). When $D$ is a (non-necessarily norm-bounded) convex subset, Theorems $3.3 \quad 3.5$ and Corollary 3.6 give several density results of the latter type.

Finally, let us point out that, in spite of density results being usually obtained under some standard constraints on the partial ordering cone $C$, throughout this paper (except in Corollary 3.2) $C$ is only assumed to be closed, convex and pointed, and no more assumptions have been made about it (about neither the existence of a base nor its compactness (of any type) or the non-emptiness of its interior).

\section{Notations and preliminary results}

Throughout this paper, $X$ will be a real normed vector space partially ordered by a closed, convex and pointed cone $C \neq\{0\}$. We denote by $X^{*}$ the dual space of $X$, by $C^{+}$the dual cone of $C$ and by $C^{+i}$ the quasi-interior of $C^{+}$i.e.,
$C^{+}=\left\{x^{*} \in X^{*}: \prec x^{*}, c \succ \geqslant 0 \forall c \in C\right\}$,

$C^{+\mathrm{i}}=\left\{x^{*} \in X^{*}: \prec x^{*}, c \succ>0 \forall c \in C \backslash\{0\}\right\}$.

It follows from the separation theorems that $C^{+}$ separates points of $X$ (i.e., for every $x \in X \backslash\{0\}$ there exists $x^{*} \in C^{+}$with $\prec x^{*}, c \succ \neq 0$; see Schaefer, 1971) and $C^{+\mathrm{i}}$ is non-empty exactly when $C$ has a base (Borwein and Zhuang, 1993).

For a subset $A \subset X$, we denote by $\operatorname{co}[A]$ the convex hull of $A$, by $\operatorname{cl}[A]$ the closure of $A$ in the norm topology, by $\sigma-\operatorname{cl}[A]$ the closure of $A$ in the weak topology $\sigma\left(X, X^{*}\right)$ and by cone $[A]$ the cone generated by $A$ (i.e., cone $[A]=\bigcup\{t A: t \geqslant 0\}$ ).

Given $x \in X$ and $\varepsilon>0$, we will denote by $B_{\varepsilon}(x)$ the closed ball with center $x$ and radius $\varepsilon$.

From now on, let $D$ be a fixed non-empty subset of $X$.

Definition 2.1. A point $x_{0} \in D$ is said to be an efficient (minimal) point of $D$ if $D \cap\left(x_{0}-C\right)=\left\{x_{0}\right\}$. A point $x_{0} \in D$ is said to be a positive proper efficient (minimal) (respectively, general positive quasi-efficient (minimal)) point of $D$ if there exists $x_{0}^{*} \in C^{+\mathrm{i}}$ (respectively, $x_{0}^{*} \in C^{+} \backslash\{0\}$ ) such that $\prec x_{0}^{*}, x_{0} \succ \leqslant \prec x_{0}^{*}, x \succ$ for all $x \in D$. We say that a point $x_{0} \in X$ is an ideal point of $D$ if there exists a closed, convex and pointed cone $K \subset X$ such that $C \subset K$ and $D \subset x_{0}+K$. If $x_{0} \in X$ is an ideal point of $D$ and $x_{0} \in D$ then we say that $x_{0}$ is a proper ideal point of $D$.

We denote the set of all efficient (minimal) points of $D$ by $\operatorname{Min}(D)$, the set of all positive proper efficient (minimal) points of $D$ by $\operatorname{Pos}(D)$, the set of all general positive quasi-efficient (minimal) points of $D$ by $\operatorname{GPos}(D)$, the set of all ideal points of $D$ by $I(D)$ and the set of all proper ideals of $D$ by $P I(D)$.

Let us provide two simple examples in order to illustrate the concepts above. Consider $R^{2}$ ordered by the usual positive cone and the convex set

$D_{1}=\left\{\left(x_{1}, x_{2}\right) \in R^{2}: 1 \leqslant x_{1}+x_{2}, 0 \leqslant x_{1} \leqslant x_{2}\right\}$.

Then,

$\operatorname{Pos}\left(D_{1}\right)=\left\{\left(x_{1}, x_{1}\right) \in R^{2}: 1=x_{1}+x_{2}, 0 \leqslant x_{1} \leqslant 1 / 2\right\}$,

$\operatorname{GPos}\left(D_{1}\right)=\operatorname{Pos}\left(D_{1}\right) \cup\left\{\left(x_{1}, x_{2}\right) \in R^{2}: x_{1}=0, x_{2} \geqslant 1\right\}$, 


$$
P I\left(D_{1}\right)=\{(0,1),(1 / 2,1 / 2)\}
$$

and

$$
\begin{aligned}
I\left(D_{1}\right)=P I\left(D_{1}\right) & \cup\left\{\left(x_{1}, x_{2}\right) \in R^{2}: x_{1}<0\right\} \\
& \cup\left\{\left(x_{1}, x_{2}\right) \in R^{2}: x_{1}+x_{2}<1\right\} \\
& \cup\left\{\left(x_{1}, x_{2}\right) \in R^{2}: x_{2}<1 / 2\right\} .
\end{aligned}
$$

For the non-convex set

$$
D_{2}=\left\{\left(x_{1}, x_{2}\right) \in R^{2}: 1 \leqslant x_{1}^{2}+x_{2}^{2}, 0 \leqslant x_{1} \leqslant x_{2}\right\}
$$

we have

$$
\operatorname{Pos}\left(D_{2}\right)=\operatorname{PI}\left(D_{2}\right)=\{(0,1),(1 / \sqrt{2}, 1 / \sqrt{2})\},
$$$$
\operatorname{GPos}\left(D_{2}\right)=\operatorname{Pos}\left(D_{2}\right) \cup\left\{\left(x_{1}, x_{2}\right) \in R^{2}: x_{1}=0, x_{2} \geqslant 1\right\}
$$

and

$$
\begin{aligned}
I\left(D_{2}\right)=P I\left(D_{2}\right) & \cup\left\{\left(x_{1}, x_{2}\right) \in R^{2}: x_{1}<0\right\} \\
& \cup\left\{\left(x_{1}, x_{2}\right) \in R^{2}:(\sqrt{2}-1) x_{1}+x_{2}<1\right\} \\
& \cup\left\{\left(x_{1}, x_{2}\right) \in R^{2}: x_{2}<1 / \sqrt{2}\right\} .
\end{aligned}
$$

From Definition 2.1 and the separation theorems, the following proposition may be stated.

Proposition 2.1. The following assertions hold:

(a) $\operatorname{PI}(D) \subset \operatorname{Min}(D) \cap I(D)$.

(b) For every $x_{0} \in I(D)$, there exist $x_{0}^{*} \in C^{+} \backslash\{0\}$ such that

$\prec x_{0}^{*}, x_{0} \succ \leqslant \prec x_{0}^{*}, x \succ \quad$ for all $x \in D$.

(c) $\operatorname{Pos}(D) \cup \operatorname{PI}(D) \subset G \operatorname{Pos}(D)$.

(d) If $C$ has non-empty interior and $D$ is convex, then $\operatorname{Min}(D) \subset G P o s(D)$.

Proposition 2.2. Let $x_{0} \in X$. The following statements are equivalent:

(a) $x_{0} \in I(D)$.

(b) There exist a real normed vector space $Y$, partially ordered by a closed convex and pointed cone $S$, and a strictly positive topological isomorphism $T: X \rightarrow Y$ such that $T(D) \subset T\left(x_{0}\right)+S$ (i.e., $T$ is a linear bijective mapping verifying that $T$ and its inverse $T^{-1}$ are both continuous, $T(C \backslash\{0\}) \subset S \backslash\{0\}$ and $\left.T(D) \subset T\left(x_{0}\right)+S\right)$.
Proof. If (a) holds, then there exists a closed, convex and pointed cone $K \subset X$ such that $C \subset K$ and $D \subset x_{0}+K$. Therefore, (b) is immediately fulfilled taking $Y=X, S=K$, and $T$ the identity map.

Suppose that (b) is verified. Then, there exist a real normed vector space $Y$, partially ordered by a closed, convex and pointed cone $S$, and a strictly positive topological isomorphism $T: X \rightarrow Y$ such that $T(D) \subset T\left(x_{0}\right)+S$. Taking $K=T^{-1}(S)$, we have that $K$ is a closed, convex and pointed cone of $X$ verifying that $C \subset K$ and $D \subset x_{0}+K$. Thus, $x_{0} \in I(D)$ and (a) holds.

Remark 2.1. Proposition 2.2 provides a necessary and sufficient condition for a point $x_{0} \in X$ to be in $I(D)$. In fact, let $\mathscr{S}$ be the set of all triples $\left(Y, Y_{+}, T\right)$, where $Y$ is a real normed vector space partially ordered by a closed, convex and pointed cone $Y_{+}$and $T: X \rightarrow Y$ is a strictly positive, surjective, linear and continuous mapping such that $T(D) \subset T\left(x_{0}\right)+Y_{+}$. For $\left(Y, Y_{+}, T\right),\left(W, W_{+}, F\right)$ $\in \mathscr{S}$, we say that $\left(Y, Y_{+}, T\right)$ precedes $\left(W, W_{+}, F\right)$ and we write $\left(Y, Y_{+}, T\right) \succsim\left(W, W_{+}, F\right)$ if there exists a positive and continuous linear function $\pi: Y \rightarrow W$ such that $\pi \circ T=F$. Consider on $\mathscr{S}$ the equivalence relation $\left(Y, Y_{+}, T\right) \approx\left(W, W_{+}, F\right)$ if and only if $\left(Y, Y_{+}, T\right) \succsim\left(W, W_{+}, F\right)$ and $\left(W, W_{+}, F\right) \succsim(Y$, $\left.Y_{+}, T\right)$. Then, the relation defined by $\left[\left(Y, Y_{+}, T\right)\right] \gg\left[\left(W, W_{+}, F\right)\right]$ if $\left(Y, Y_{+}, T\right) \succsim\left(W, W_{+}\right.$, $F)$ is an order relation on the quotient set $\mathscr{S} / \approx$. If $\mathscr{S} \neq \emptyset$ (in particular, if $x_{0} \in \operatorname{Pos}(D)$ ), it may be easily proved that there exists an element in $\mathscr{S} / \approx$ which precedes all the elements of $\mathscr{S} / \approx$ (i.e., there exists a maximum of $(\mathscr{S} / \approx, \gg)$ ), and it results now from Proposition 2.2 that $x_{0} \in I(D)$ if and only if the maximum element $\left[\left(Y, Y_{+}, T\right)\right]$ of $(\mathscr{S} / \approx, \gg)$ verifies that $T$ is a topological isomorphism (it also may be easily proved that $T$ being a topological isomorphism does not depend on the choice of the representative element of the equivalence class).

If $x_{0} \in I(D)$, then it trivially follows that the maximum element of $(\mathscr{S} / \approx, \gg)$ is $[(X, L, I)]$, where $I$ denotes the identity map, and $L$ is the intersection of all closed, convex and pointed cones $K \subset X$ such that $C \subset K$ and $D \subset x_{0}+K$. 
Proposition 2.3 below provides a sufficient condition for $x_{0} \in X$ to be an ideal point of $D$. This condition may be very easily tested in practice and will be very useful in future sections.

Proposition 2.3. Let $x_{0} \in X$. If the set

$C^{+}\left(x_{0}, D\right)=\left\{x^{*} \in C^{+}: \prec x^{*}, x_{0} \succ \leqslant \prec x^{*}, x \succ \forall x \in D\right\}$

separates points of $X$, then $x_{0} \in I(D)$.

Proof. The set $C^{++}\left(x_{0}, D\right)=\left\{x \in X: \prec x^{*}, x \succ \geqslant\right.$ $\left.0 \forall x^{*} \in C^{+}\left(x_{0}, D\right)\right\}$ is clearly a closed convex cone verifying that $C \subset C^{++}\left(x_{0}, D\right)$ and $D \subset x_{0}+$ $C^{++}\left(x_{0}, D\right)$. Furthermore, $C^{++}\left(x_{0}, D\right)$ is also pointed because if $x \in C^{++}\left(x_{0}, D\right) \cap\left(-C^{++}\left(x_{0}, D\right)\right)$ then $\prec x^{*}, x \succ=0$ for all $x^{*} \in C^{+}\left(x_{0}, D\right)$, and therefore $x=0$ since $C^{+}\left(x_{0}, D\right)$ separates points of $X$. It follows now from Definition 2.1 that $x_{0} \in I(D)$.

We present now a sufficient condition for a point $x_{0} \in D$ to be a proper ideal point of $D$.

Proposition 2.4. Let $x_{0} \in D$. If $0 \notin \mathrm{cl}[\mathrm{co}[$ (cone $\left.\left.\left.\left(D-x_{0}\right)+C\right) \backslash B_{\varepsilon}(0)\right]\right]$ for some $\varepsilon>0$, then $x_{0} \in P I(D)$.

Proof. Let $\varepsilon>0$ be such that

$0 \notin \operatorname{cl}\left[\operatorname{co}\left[\left(\operatorname{cone}\left(D-x_{0}\right)+C\right) \backslash B_{\varepsilon}(0)\right]\right]$.

Then

$$
\begin{array}{r}
0 \notin \mathrm{cl}\left[\operatorname { c o } \left[\left(\operatorname{cone}\left(D-x_{0}\right)+C\right)\right.\right. \\
\left.\left.\cap\left(B_{2 \varepsilon}(0) \backslash B_{\varepsilon}(0)\right)\right]\right] .
\end{array}
$$

Define

$K=\operatorname{cl}[\operatorname{cone}(A)]$.

with

$A=\operatorname{co}\left[\left(\operatorname{cone}\left(D-x_{0}\right)+C\right) \cap\left(B_{2 \varepsilon}(0) \backslash B_{\varepsilon}(0)\right)\right]$.

Then $K$ is obviously a closed convex cone such that $C \subset K$ and $D-\left\{x_{0}\right\} \subset K$. Therefore, if $K$ is also pointed, then $x_{0} \in I(D)$ and the proof will be finished. Thus, let us prove that the cone $K$ is pointed. In fact, suppose the existence of $x \in X \backslash\{0\}$ such that $x \in K \cap(-K)$. Then, there exist $\left(a_{n}\right)_{n \in N},\left(b_{n}\right)_{n \in N} \subset A$ and $\left(t_{n}\right)_{n \in N},\left(s_{n}\right)_{n \in N} \subset R_{+}$ such that $x=\lim _{n \rightarrow \infty} t_{n} a_{n}$ and $-x=\lim _{n \rightarrow \infty} s_{n} b_{n}$. Without loss of generality (taking subsequences if necessary) we may assume the existence of $0 \leqslant t, s \leqslant \infty \quad$ such that $t=\lim _{n \rightarrow \infty} t_{n}$ and $s=\lim _{n \rightarrow \infty} s_{n}$. Since $\|x\|=\lim _{n \rightarrow \infty} t_{n}\left\|a_{n}\right\|$, we have $t<\infty$, because otherwise $\left(a_{n}\right)_{n \in N}$ tends to zero and this contradicts (1). Furthermore, since $t<\infty$ and $\left\|a_{n}\right\| \leqslant 2 \varepsilon$ for all $n \in N$, we have

$x=\lim _{n \rightarrow \infty} t_{n} a_{n}=\lim _{n \rightarrow \infty} t a_{n}$.

Therefore, $t \neq 0$ since $x \in X \backslash\{0\}$. Thus, $0<t<\infty$.

In an analogous way it may be proved that $0<s<\infty$ and

$-x=\lim _{n \rightarrow \infty} s b_{n}$.

It follows from (2) and (3) that

$$
\begin{aligned}
0 & =\lim _{n \rightarrow \infty}\left(t a_{n}+s b_{n}\right) \\
& =(t+s) \lim _{n \rightarrow \infty}\left(\frac{t}{t+s} a_{n}+\frac{s}{t+s} b_{n}\right) .
\end{aligned}
$$

The last expression contradicts (1) because $\left(t /(t+s) a_{n}+s /(t+s) b_{n}\right) \in A$ for all $n \in N$.

Therefore, there is no $x \in X \backslash\{0\}$ such that $x \in K \cap(-K)$ and the cone $K$ is pointed.

Remark 2.2. The assumption in Proposition 2.4 trivially holds if 0 is a denting point for cone $\left(D-x_{0}\right)+C$ (recall that for any subset $A \subset X$ a point $x \in A$ is said to be a denting point for $A$ if, for each $\varepsilon>0, \quad x \notin \operatorname{cl}\left[\operatorname{co}\left[A \backslash B_{\varepsilon}(x)\right]\right]$; see Gong, 1995, or Diestel and Uhl, 1977).

\section{Density theorems}

In this section, we state some results concerning the density of $I(D)$ in the efficient line.

Theorem 3.1. If the set $D$ is norm-bounded, then $G \operatorname{Pos}(D) \subset \operatorname{cl}[I(D)]$. 
Proof. Let $x_{0} \in G P o s(D)$ and $\varepsilon>0$. Then, there exists $x_{0}^{*} \in C^{+} \backslash\{0\}$ verifying $\prec x_{0}^{*}, x_{0} \succ \leqslant \prec x_{0}^{*}$, $x \succ$ for all $x \in D$. Since $x_{0}^{*} \neq 0$, we can find $x_{1} \in X$ such that $\left\|x_{1}\right\|=1$ and $\prec x_{0}^{*}, x_{1} \succ<0$. Define $x_{\varepsilon}=x_{0}+\varepsilon x_{1}$ and $\alpha=-\prec x_{0}^{*}, x_{1} \succ$. Then, $\left\|x_{0}-x_{\varepsilon}\right\|=\varepsilon$ and

$\prec x_{0}^{*}, x_{\varepsilon} \succ+\alpha \varepsilon \leqslant \prec x_{0}^{*}, x \succ$

for all $x \in D$. Furthermore, since $D$ is normbounded, there exists $r>0$ such that

$\left|\prec x^{*}-x_{0}^{*}, x \succ\right| \leqslant \frac{\alpha \varepsilon}{2}$

and

$\left|\prec x^{*}-x_{0}^{*}, x_{\varepsilon} \succ\right| \leqslant \frac{\alpha \varepsilon}{2}$

for all $x \in D$ and $x^{*} \in X^{*}$ with $\left\|x^{*}-x_{0}^{*}\right\|<r$.

Now, it results from (4) (6) that

$$
\begin{aligned}
\prec x^{*}, x \succ & \geqslant \prec x_{0}^{*}, x \succ-\frac{\alpha \varepsilon}{2} \\
& \geqslant \prec x_{0}^{*}, x_{\varepsilon} \succ+\frac{\alpha \varepsilon}{2} \\
& \geqslant \prec x^{*}, x_{\varepsilon} \succ
\end{aligned}
$$

for all $x \in D$ and $x^{*} \in X^{*}$ with $\left\|x^{*}-x_{0}^{*}\right\|<r$.

Furthermore, it follows from (7) that

$$
\begin{aligned}
B_{r}^{*} & =\left\{x^{*} \in C^{+}:\left\|x^{*}-x_{0}^{*}\right\|<r\right\} \subset C^{+}\left(x_{\varepsilon}, D\right) \\
& =\left\{x^{*} \in C^{+}: \prec x^{*}, x_{\varepsilon} \succ \leqslant \prec x^{*}, x \succ \forall x \in D\right\} .
\end{aligned}
$$

Therefore, since $C^{+}$separates points of $X$, so does $B_{r}^{*}$, and consequently, $C^{+}\left(x_{\varepsilon}, D\right)$ separates points of $X$ too. It results now from Proposition 2.3 that $x_{\varepsilon} \in I(D)$, and the proof is finished because $\left\|x_{\varepsilon}-x_{0}\right\|=\varepsilon$.

Corollary 3.2. Suppose that $C$ has non-empty interior. If the set $D$ is norm-bounded and convex, then $\operatorname{Min}(D) \subset \operatorname{cl}[I(D)]$.

Proof. It is an immediate consequence of statement (d) in Proposition 2.1 and Theorem 3.1.

When $D$ is a (non-necessarily norm-bounded) convex subset of $X$, we have the following results.
Theorem 3.3. Let $D$ be a convex subset of $X$ and $x_{0} \in D$. Suppose that $D(t)=D \cap B_{t}\left(x_{0}\right)$ is compact for some $t>0$, and there exists $x_{0}^{*} \in C^{+} \backslash\{0\}$ such that $\prec x_{0}^{*}, x_{0} \succ<\prec x_{0}^{*}, x \succ$ for all $x \in D \backslash\left\{x_{0}\right\}$. Then $x_{0} \in \operatorname{cl}[I(D)]$.

Proof. Let $x_{0}^{*} \in C^{+} \backslash\{0\}$ be such that $\prec x_{0}^{*}, x_{0} \succ<\prec x_{0}^{*}, x \succ$ for all $x \in D$. For every $\varepsilon>0$, let us define $x_{\varepsilon}=x_{0}+\varepsilon x_{1}$ and $\alpha=-\prec x_{0}^{*}, x_{1} \succ$, where $x_{1} \in X$ is such that $\left\|x_{1}\right\|=1$ and $\prec x_{0}^{*}, x_{1} \succ<0$ (such an element exists because $\left.x_{0}^{*} \neq 0\right)$. Then $\left\|x_{0}-x_{\varepsilon}\right\|=\varepsilon$ and

$\prec x_{0}^{*}, x_{\varepsilon} \succ+\alpha \varepsilon \leqslant \prec x_{0}^{*}, x \succ$

for all $x \in D$.

Let $D_{n}=D \cap B_{n}\left(x_{\varepsilon}\right)$ for all $n \in N$. Then, $D_{n}$ is norm-bounded and thus, there exists $r_{n}>0$ such that the sequence $\left(r_{n}\right)_{n=1}^{\infty}$ tends to zero and

$\left|\prec x^{*}-x_{0}^{*}, x \succ\right| \leqslant \frac{\alpha \varepsilon}{2} \quad$ and

$\left|\prec x^{*}-x_{0}^{*}, x_{\varepsilon} \succ\right| \leqslant \frac{\alpha \varepsilon}{2}$

for all $x \in D$ and $x^{*} \in X^{*}$ with $\left\|x^{*}-x_{0}^{*}\right\|<r_{n}$.

It follows now from (8) and (9) that

$$
\begin{aligned}
\prec x^{*}, x \succ & \geqslant \prec x_{0}^{*}, x \succ-\frac{\alpha \varepsilon}{2} \geqslant \prec x_{0}^{*}, x_{\varepsilon} \succ \\
+\frac{\alpha \varepsilon}{2} & \geqslant \prec x^{*}, x_{\varepsilon} \succ
\end{aligned}
$$

for all $x \in D_{n}$ and $x^{*} \in X^{*}$ with $\left\|x^{*}-x_{0}^{*}\right\|<r_{n}$.

Therefore,

$$
\begin{aligned}
B_{r_{n}}^{*} & =\left\{x^{*} \in C^{+}:\left\|x^{*}-x_{0}^{*}\right\|<r_{n}\right\} \subset C^{+}\left(x_{\varepsilon}, D_{n}\right) \\
& =\left\{x^{*} \in C^{+}: \prec x^{*}, x_{\varepsilon} \succ \leqslant \prec x^{*}, x \succ \quad \forall x \in D_{n}\right\}
\end{aligned}
$$

for all $n \in N$, and

$$
\begin{aligned}
\bigcap_{n=1}^{\infty} C^{+}\left(x_{\varepsilon}, D_{n}\right)= & C^{+}\left(x_{\varepsilon}, D\right) \\
= & \left\{x^{*} \in C^{+}: \prec x^{*}, x_{\varepsilon} \succ\right. \\
& \left.\leqslant \prec x^{*}, x \succ \forall x \in D\right\} .
\end{aligned}
$$

The proof may be finished just proceeding as in the proof of Theorem 3.1, if there exists $m \in N$ such that 
$B_{r_{m}}^{*} \subset \bigcap_{n=1}^{\infty} C^{+}\left(x_{\varepsilon}, D_{n}\right)=C^{+}\left(x_{\varepsilon}, D\right)$.

Thus, let us prove the existence of $m$. If $r_{n}$ does not verify (13) for all $n \in N$, then we can find two sequences $\left(x_{n}^{*}\right)_{n \in N} \subset C^{+}$and $\left(x_{n}\right)_{n \in N} \subset D$, such that $\left(x_{n}^{*}\right)_{n \in N}$ tends to $x_{0}^{*}$ (in the norm topology on $X^{*}$ ) and

$\prec x_{n}^{*}, x_{n} \succ<\prec x_{n}^{*}, x_{\varepsilon} \succ$,

$\prec x_{n}^{*}, x_{\varepsilon} \succ \leqslant \prec x_{n}^{*}, x_{0} \succ$

and

$\left\|x_{n}-x_{0}\right\|>n$

for all $n \in N$.

It results now from (16) that there exists $n_{t} \in N$ such that $t_{n}=\left(t /\left\|x_{n}-x_{0}\right\|\right) \in(0,1)$ for all $n \geqslant n_{t}$. Therefore, $d_{n}=t_{n} x_{n}+\left(1-t_{n}\right) x_{0} \in D$ for all $n \geqslant n_{t}$ and $d_{n} \in D(t)$ because

$\left\|d_{n}-x_{0}\right\|=t_{n}\left\|x_{n}-x_{0}\right\|=t$.

Since $\left(d_{n}\right)_{n \geqslant n_{t}} \subset D(t)$ and $D(t)$ is compact, the sequence $\left(d_{n}\right)_{n \geqslant n_{t}}$ has (at least) an adherent point $d \in D(t) \subset D$.

Furthermore, (14) and (15) imply that

$$
\begin{aligned}
\prec x_{n}^{*}, d_{n} \succ & =t_{n} \prec x_{n}^{*}, x_{n} \succ+\left(1-t_{n}\right) \prec x_{n}^{*}, x_{0} \succ \\
& \leqslant t_{n} \prec x_{n}^{*}, x_{\varepsilon} \succ+\left(1-t_{n}\right) \prec x_{n}^{*}, x_{0} \succ \\
& \leqslant \prec x_{n}^{*}, x_{0} \succ .
\end{aligned}
$$

And, since $\left(x_{n}^{*}\right)_{n \in N}$ tends to $x_{0}^{*}$ (in the norm topology on $X^{*}$ ), we also have

$\prec x_{0}^{*}, x_{0} \succ=\lim _{n \rightarrow \infty} \prec x_{n}^{*}, x_{0} \succ$.

It follows now from (18) and (19) that

$\prec x_{0}^{*}, d \succ=\lim _{n \rightarrow \infty} \prec x_{n}^{*}, d \succ \leqslant \prec x_{0}^{*}, x_{0} \succ$.

The proof is already finished because the latter expression contradicts the hypotheses since (17) implies $\left\|d-x_{0}\right\|=t>0$.

Theorem 3.4. Let $D$ be a convex subset of $X$ such that the intersection of $D$ with every closed ball in $X$ is compact. Let $x_{0} \in D$, and suppose that there exists $x_{0}^{*} \in C^{+} \backslash\{0\}$ such that $\prec x_{0}^{*}, x_{0} \succ \leqslant \prec x_{0}^{*}, x \succ$ for all $x \in D$, and the subset $\left\{x \in D: \prec x_{0}^{*}, x \succ=\prec x_{0}^{*}, x_{0} \succ\right\}$ is norm-bounded. Then $x_{0} \in \mathrm{cl}[I(D)]$.

Proof. Let $x_{\varepsilon}$ be as in the proof of Theorem 3.3 for $\varepsilon>0$. If $x_{0} \notin \mathrm{cl}[I(D)]$ there exists $\varepsilon>0$ such that $x_{\varepsilon} \notin I(D)$. Then, proceeding as in the proof of Theorem 3.3, we find $d_{n} \in D(n)=D \cap B_{n}\left(x_{0}\right)$, such that

$\left\|d_{n}-x_{0}\right\|=n \quad$ and $\quad \prec x_{0}^{*}, d_{n} \succ \leqslant \prec x_{0}^{*}, x_{0} \succ$

for every $n \in N$. Therefore, $\left(d_{n}\right)_{n \in N} \subset\{x \in D$ : $\left.\prec x_{0}^{*}, x \succ=\prec x_{0}^{*}, x_{0} \succ\right\}$ and we have a contradiction.

Theorem 3.5. Let $D$ be a convex subset of $X$ and $x_{0} \in D$. Suppose that $D(t)=D \cap B_{t}\left(x_{0}\right)$ is $\sigma\left(X, X^{*}\right)$ compact for some $t>0$, and there exist $x_{0}^{*} \in C^{+} \backslash\{0\}$ and $0<\lambda<t$ such that $\prec x_{0}^{*}, x_{0} \succ$ $<\prec x_{0}^{*}, x \succ$ for all $x \in D \backslash\left\{x_{0}\right\}$ and $x_{0} \notin \mathrm{cl}$ $\left[\operatorname{co}\left[D \backslash B_{\lambda}\left(x_{0}\right)\right]\right]$. Then $x_{0} \in \operatorname{cl}[I(D)]$.

Proof. It is enough to proceed as in the proof of Theorem 3.3, remarking that now $d$ is a $\sigma\left(X, X^{*}\right)$ adherent point of $\left(d_{n}\right)_{n \geqslant n_{t}}$, and (17) does not imply necessarily that $d \in D \backslash\left\{x_{0}\right\}$. In this case the expression $d \in D \backslash\left\{x_{0}\right\}$ follows from the hypotheses, since the closed convex subsets of $X$ for the norm and the weak topologies coincide (see Proposition 3.4.3 in Horvath, 1966), and therefore

$$
\begin{aligned}
& d \in \sigma-\operatorname{cl}\left[D \backslash B_{\lambda}\left(x_{0}\right)\right] \\
& \quad \subset \sigma-\operatorname{cl}\left\{\operatorname{co}\left[D \backslash B_{\lambda}\left(x_{0}\right)\right]\right\}=\operatorname{cl}\left\{\operatorname{co}\left[D \backslash B_{\lambda}\left(x_{0}\right)\right]\right\} .
\end{aligned}
$$

Corollary 3.6. Let $D$ be a convex subset of $X$ and $x_{0} \in D$. If there exists $t>0$ such that $D(t)=D \cap B_{t}\left(x_{0}\right) \quad$ is $\quad \sigma\left(X, X^{*}\right)$-compact, and $x_{0} \notin \operatorname{cl}\left[\operatorname{co}\left[(D+C) \backslash B_{\lambda}\left(x_{0}\right)\right]\right]$ for some $0<\lambda<t$, then $x_{0} \in \operatorname{cl}[I(D)]$.

Proof. It follows from Theorem 3.5 and the second separation theorem (Schaefer, 1971).

Remark 3.1. The assumptions about the compactness of $D(t)$ for some $t>0$, in Theorem 3.3, and about the compactness of the intersection of $D$ with every closed ball in $X$, in Theorem 3.4, are automatically verified if $X=R^{n}$ and the subset $D$ is 
closed. Furthermore, the assumption about the $\sigma\left(X, X^{*}\right)$-compactness of $D(t)$ for some $t>0$, in Theorem 3.5 and Corollary 3.6, is trivially fulfilled if the space $X$ is reflexive and the subset $D$ is $\sigma\left(X, X^{*}\right)$-closed. Note also that the assumption about the existence of $\lambda \in(0, t)$ such that

$x_{0} \notin \mathrm{cl}\left[\operatorname{co}\left[D \backslash B_{\lambda}\left(x_{0}\right)\right]\right]$

(respectively, $\left.x_{0} \notin \mathrm{cl}\left[\operatorname{co}\left[(D+C) \backslash B_{\lambda}\left(x_{0}\right)\right]\right]\right)$,

in Theorem 3.5 (respectively, in Corollary 3.6), is satisfied if $x_{0}$ is a denting point of $D$ (respectively, of $D+C$ ). Finally, let us remark that assumptions made in Theorems 3.3 3.5, and Corollary 3.6, trivially imply that $x_{0} \in G P o s(D)$.

\section{Conclusions}

If $D$ is a norm-bounded convex subset of $X$, Corollary 3.2 states that the set of ideal points in $D$ is dense in the set of minimal points of $D$ if the closed, convex and pointed cone $C$ has non-empty interior. Nevertheless, since $\operatorname{Pos}(D) \subset G \operatorname{Pos}(D)$, results of this kind may be also obtained without this assumption, by combining Theorem 3.1 with some extensions to normed vector spaces of the Arrow Barankin Blackwell theorem. Something similar may be done when $D$ is a (non-necessarily norm-bounded) convex subset of $X$, using Theorems 3.3 3.5, and Corollary 3.6. Finally, let us point out that the usefulness of density results for ideal points in the efficient line is clear, since most questions in vector optimization (sensitivity analysis, for instance) have an easier treatment for minimum solutions than for minimal ones.

\section{Acknowledgements}

This research was partially supported by CAM, project on Complex Decision Problems, and by D.G.I.C.Y.T., project PB95-0729-C02-02.

\section{References}

Arrow, K.J., Barankin, E.W., Blackwell, D., 1953. Admissible points of convex sets. In: Kuhn, H.W., Tucker, A.W. (Eds.), Contribution to the Theory of Games. Princeton University Press, Princeton, NJ, pp. 8792.

Balbás, A., Fernández, F., Jiménez Guerra, P., 1995. On the envolvent theorem in multiobjective programming. Indian Journal of Pure and Applied Mathematics 26, 10351047.

Balbás, A., Jiménez Guerra, P., 1996. Sensitivity analysis for convex multiobjective programming in abstract spaces. Journal of Mathematical Analysis and Applications 202, 645658.

Balbás, A., Jiménez Guerra, P., 1997. Sensitivity in multiob jective programming by differential equations methods. The case of homogeneous functions. In: Lecture Notes in Economics and Mathematical Systems, vol. 455. Springer, Berlin, pp. 188196.

Borwein, J.M., Zhuang, D., 1993. Super efficiency in vector optimization. Transactions of the American Mathematical Society 338, 105122.

Chen, G.Y., 1995. Generalized Arrow Barankin Blackwell theorems in locally convex spaces. Journal of Optimization Theory and Applications 84, 93101.

Diestel, J., Uhl, J.J., 1977. Vector measures. Mathematical Surveys and Monographies No. 5, American Mathematical Society, Providence, RI.

Ferro, F., 1993. General form of the Arrow Barankin Black well theorem in normed spaces and the $l^{\infty}$ case. Journal of Optimization Theory and Applications 79, 127138.

Gallagher, R.J., Saleh, O.A., 1993. Two generalizations of a theorem of Arrow, Barankin and Blackwell. SIAM Journal of Control and Optimization 31, 247256.

Gong, X.H., 1995. Density of the set of positive proper minimal points in the set of minimal points. Journal of Optimization Theory and Applications 86, 609630.

Horvath, J., 1966. Topological Vector Spaces and Distribu tions, vol 1. Addison Wesley, Reading, MA.

Makarov, E.K., Rachkovski, N.N., 1996. Density theorems for generalized proper efficiency. Journal of Optimization Theory and Applications 91, 419437.

Schaefer, H.H., 1971. Topological Vector Spaces. Springer, Berlin.

Zowe, S., 1977. The saddle point theorem of Kuhn and Tucker in ordered vector spaces. Journal of Mathematical Analysis and Applications 57, 4155. 